\title{
OPTICAL SENSORS
}

\section{Checking cell temperature}

A new optical technique for accurately mapping the temperature distribution within single cells looks set to provide important insights into their thermodynamics and aid the development of photothermal cancer therapies (Nano Lett. 12, 2107-2111; 2012)

The scheme, developed by scientists from the Institute of Photonic Sciences (ICFO) and the Catalan Institution for Research and Advanced Studies (ICREA) in Barcelona, Spain, and the Institut Fresnel in Marseille, France, makes use of green fluorescent protein (GFP) as a thermal probe. Temperature measurements are made by monitoring the polarization anisotropy of fluorescence from GFP, which is known to be temperature-dependent. As the temperature rises, increased Brownian rotational motion of the cells reduces the polarization anisotropy of their light emission.

"As with most chemical reactions, many intercellular processes such as gene expression and cell division, for example, are exothermic," explained Romain Quidant, a researcher from ICFO involved in the study. "The possibility of measuring intracellular temperature could be the basis of the development of a quite

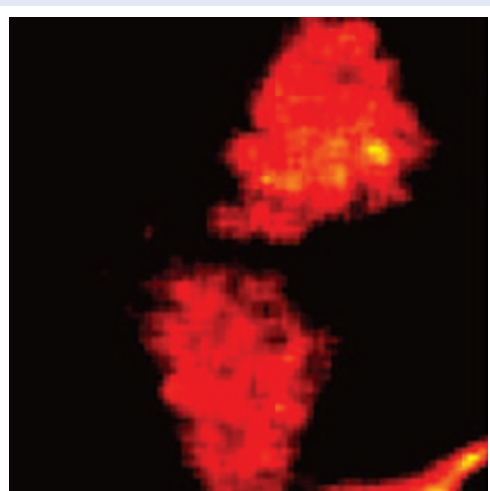

unexplored field: thermal biology at the single-cell level."

The researchers tested their technique with HeLa and U-87 MG cancer cells that had been transfected with GFP and were surrounded by photothermally heated gold nanorods. The study employed a confocal microscope equipped with two laser sources: an infrared laser for heating the nanorods and a blue laser to excite the GFP. Results showed that intracellular measurements with a spatial resolution of $300 \mathrm{~nm}$ and a temperature accuracy of $0.4{ }^{\circ} \mathrm{C}$ are possible. The approach is also non-invasive, provides fast read-out and allows data

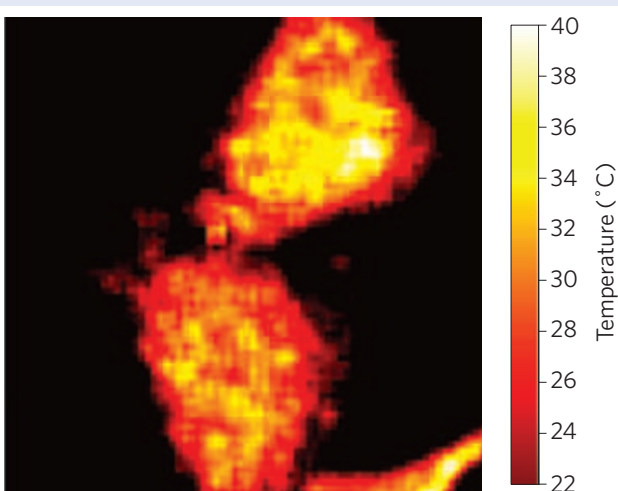

to be collected with a time resolution of $20 \mathrm{~ms}$.

The researchers are now investigating strategies for improving the accuracy and resolution of the method and are thinking of applying it to in vivo measurements.

"The intracellular temperature mapping of single cells will help to explain the thermodynamics of different cell organelles," commented Quidant. "This information could be extremely important to monitor and thus further understand, for example, the increased production of heat of cancer cells over normal cells."

\section{OLIVER GRAYDON}

\section{LASER PARTICLE ACCELERATION}

\section{Pushing atoms with light}

Controlling the velocity of neutral particles is an experimental challenge, owing to their absence of charge.

Scientists have now demonstrated a technique that can be used to accelerate neutral argon atoms by polarizing them in moving optical lattices.

\section{Andreas Osterwalder}

ontrol over the internal and external degrees of freedom of atoms or

molecules in the gaseous phase is a much-anticipated goal for spectroscopy, dynamics studies and even solid-state physics. Although there has been tremendous progress towards achieving this goal over the past decade, applications are often limited to polar or paramagnetic molecules ${ }^{1}$.

Writing in Nature Photonics, Barker and co-workers now introduce a new method that uses an accelerating optical lattice to accelerate or decelerate polarizable particles ${ }^{2}$. The electric field maxima in the lattice are potential minima that drag polarizable particles along as the lattice moves and accelerates. This scheme is shown in Fig. 1: the two counterpropagating laser beams (red) produce a moving optical lattice. The lattice extracts atoms from a cloud that is initially at rest and accelerates them towards a detector.

Control over the transverse motion of neutral atoms in a magnetic field was achieved by Stern and Gerlach ${ }^{3}$ in 1922 , and the use of electric fields to control polar molecules was proposed shortly thereafter ${ }^{4}$. However, control over the forward motion of neutral atoms, through interactions with laser beams, was demonstrated only 3-4 decades ago - an achievement that was honoured by the Nobel Prize for physics in 1997. Laser cooling was a revolutionary technique that propelled the entire field of atomic physics into a new era. It allowed atoms to be trapped and cooled to 